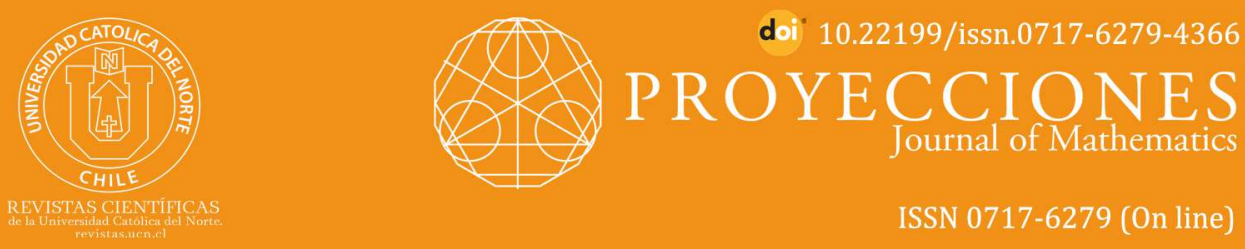

\title{
Lyapunov-type inequality for higher order left and right fractional $\mathrm{p}$-Laplacian problems
}

\section{Alberto Cabada1 ${ }^{1}$ orcid.org/0000-0003-1488-935X Rabah Khaldi² ${ }^{2}$ orcid.org/0000-0002-6462-4424}

${ }^{1}$ Universidade de Santiago de Compostela, Dept. de Estatística, Análise Matemática e Optimización, Santiago de Compostela, Galicia, Spain.

alberto.cabada@usc.gal

${ }^{2}$ Badji Mokhtar Annaba University, Dept. of Mathematics, Annaba, Algeria.

-

Received: 2020/07/29 | Accepted: 2021/02/20

\section{Abstract:}

In this paper, we consider a $p$-Laplacian eigenvalue boundary value problem involving both right Caputo and left Riemann-Liouville types fractional derivatives. To prove the existence of solutions, we apply the Schaefer's fixed point theorem. Furthermore, we present the Lyapunov inequality for the corresponding problem.

Keywords: Fractional calculus; Lyapunov inequality; p-Laplacian operator; Eigenvalue problem.

MSC (2020): 74H20, 26D15, 34A08.

\section{Cite this article as (IEEE citation style):}

A. Cabada and R. Khaldi, "Lyapunov-type inequality for higher order left and right fractional p-Laplacian problems", Proyecciones (Antofagasta, On line), vol. 40, no. 4, pp. 1031-1040, 2021, doi: 10.22199/issn.0717-6279-4366

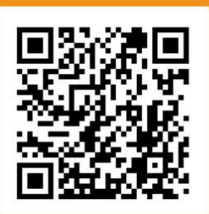

Article copyright: (C) 2021 Alberto Cabada and Rabah Khaldi. This is an open access article distributed under the terms of the Creative Commons License, which permits unrestricted use and distribution provided the original author and source are credited. 


\section{Introduction}

In this work, firstly, we prove the existence of solutions for an iterated fractional boundary value problem

$$
\left\{\begin{array}{c}
{ }^{C} D_{1^{-}}^{\alpha}\left(\phi_{p}\left(D_{0^{+}}^{\beta} u(t)\right)\right)+\lambda f(t, u(t))=0,0<t<1 \\
u^{(i)}(0)=D_{0^{+}}^{\beta+i} u(1)=0, i=0,1, \ldots, n-1,
\end{array}\right.
$$

then, we obtain Lyapunov type inequalities for the corresponding problem

$$
\left\{\begin{array}{c}
-{ }^{C} D_{b^{-}}^{\alpha}\left(\phi_{p}\left(D_{a^{+}}^{\beta} u(t)\right)\right)+\chi(t) \phi_{p}(u(t))=0, a<t<b \\
u^{(i)}(a)=D_{a^{+}}^{\beta+i} u(b)=0, i=0,1, \ldots, n-1,
\end{array}\right.
$$

where $n-1<\alpha, \beta \leq n, n \geq 2,{ }^{C} D_{b^{-}}^{\alpha}$ and $D_{a^{+}}^{\beta}$ refer to the right Caputo derivative and the left Riemann-Liouville derivative respectevely, $\phi_{p}(s)=$ $s|s|^{p-2}, p>2, \phi_{p}^{-1}=\phi_{q}, \frac{1}{p}+\frac{1}{q}=1$ and $\chi:[a, b] \rightarrow \mathbf{R}$ is a continuous function.

The problems generated by equations involving both left and right fractional derivatives, arise in the study of Euler-Lagrange equations for fractional problems of calculus of variations, see $[4,14,15]$. Recently, this type of problems has been considered by many authors, see $[1,2,3,5,6$, $7,8,9,10,12,16]$.

Concerning the Lyapunov inequality and its generalizations, it has been shown to be very useful in different problems, such as oscillation, asymptotic theory, disconjugacy and eigenvalue problems. For results on Lyapunov inequality one may suggest the papers $[7,10,11,17]$.

The paper is organized as follows. In Section 2, we briefly recall some essential definitions and lemmas on fractional calculus to be used later. Our results are formulated and proved in Sections 3 and 4. The main results are Theorem 2, which establishes existence of solution for the eigenvalue problem for left and right fractional differential equation 1.1, and Theorem t-lyapunov where, we obtain a new Lyapunov type inequality for problem 1.2 .

\section{Basic results}

In this section, we recall some essential definitions and preliminary facts that will be used in the sequel and can be found in $[13,18,19]$.

Definition 1. The left and right fractional integrals of order $\alpha>0$ of a function $g \in L^{1}[a, b]$ are defined respectively by 


$$
\begin{aligned}
I_{a^{+}}^{\alpha} g(t) & =\frac{1}{\Gamma(\alpha)} \int_{a}^{t}(t-s)^{\alpha-1} g(s) d s, \\
I_{b^{-}}^{\alpha} g(t) & =\frac{1}{\Gamma(\alpha)} \int_{t}^{b}(s-t)^{\alpha-1} g(s) d s .
\end{aligned}
$$

Definition 2. The left and right Riemann-Liouville fractional derivatives of order $n-1<\alpha \leq n, n \geq 1$ of a function $g \in A C^{n}[a, b]$ are defined respectively by

$$
\begin{aligned}
D_{a^{+}}^{\alpha} g(t) & =\frac{d^{n}}{d t^{n}}\left(I_{a^{+}}^{n-\alpha} g\right)(t), \\
D_{b^{-}}^{\alpha} g(t) & =(-1)^{n} \frac{d^{n}}{d t^{n}}\left(I_{a^{+}}^{n-\alpha} g\right)(t) .
\end{aligned}
$$

Definition 3. The left and right Caputo fractional derivatives of order $n-1<\alpha \leq n, n \geq 1$ of a function $g \in A C^{n}[a, b]$ are defined respectively by

$$
\begin{aligned}
{ }^{C} \mathcal{D}_{a^{+}}^{\alpha} g(t) & =I_{d^{-}}^{n-\alpha} g^{(n)}(t), \\
{ }^{C} \mathcal{D}_{b^{-}}^{\alpha} g(t) & =(-1)^{n} I_{d^{-}}^{n-\alpha} g^{(n)}(t) .
\end{aligned}
$$

With respect to the composition rule of fractional operators, we mention the following.

Proposition 4. Let $\alpha \in(n-1, n]$ and $f \in L_{1}[a, b]$. Then

$$
\begin{aligned}
& I_{a^{+}}^{\alpha C} D_{a^{+}}^{\alpha} f(t)=f(t)-\sum_{k=0}^{n-1} \frac{f^{(k)}(a)}{k !}(t-a)^{k}, \\
& I_{b^{-}}^{\alpha C} D_{b^{-}}^{\alpha} f(t)=f(t)-\sum_{k=0}^{n-1} \frac{(-1)^{k} f^{(k)}(b)}{k !}(b-t)^{k} .
\end{aligned}
$$

Next, we cite Schaefer's fixed point theorem:

Theorem 5. [19]. Let $T$ be a continuous and compact mapping of a Banach space $X$ into itself, such that the set

$\{\mathrm{x} \in X: x=\mu T x$ for some $0 \leq \mu \leq 1\}$ is bounded. Then $T$ has a fixed point. 


\section{Existence of solutions}

In this section, we prove the existence of solutions for the eigenvalue problem 1.1 .

First, we begin by solving the following linear fractional problem:

$$
\left\{\begin{array}{l}
D_{0^{+}}^{\beta} u(t)+y(t)=0,0<t<1 \\
u^{(i)}(0)=0, i=0,1, \ldots, n-1 .
\end{array}\right.
$$

Lemma 1. For $n-1<\beta \leq n, n \geq 2$, the solution of problem 1.3 is given by

$$
u(t)=-\frac{1}{\Gamma(\beta)} \int_{0}^{t}(t-s)^{\beta-1} y(s) d s .
$$

Proof. We get (3.2) by applying (2.1) in (3.1) and using the boundary conditions $u^{(i)}(0)=0, i=0,1, \ldots, n-1$.

Now, we transform the boundary value problem to an integral equation.

Lemma 2. A function $u$ is a solution of the linear boundary value problem 1.1 if and only if $u$ satisfies the integral equation

$$
u(t)=\frac{1}{(\Gamma(\alpha))^{q-1} \Gamma(\beta)} \int_{0}^{t}(t-s)^{\beta-1} \phi_{q}\left(\int_{s}^{1}(r-s)^{\alpha-1} \lambda f(r, u(r)) d r\right) d s .
$$

Proof. Applying the operator $I_{1^{-}}^{\alpha}$ on both sides of the differential equation in 1.1 and using (2.2) together with the boundary conditions $D_{0^{+}}^{\beta-i} u(1)=0, i=0,1, \ldots, n-1$, then the boundary value problem 1.1 reduces to the following problem

$$
\begin{aligned}
& \left\{\begin{array}{c}
D_{0^{+}}^{\beta} u(t)-\phi_{q}\left(\frac{1}{\Gamma(\alpha)} \int_{t}^{1}(s-t)^{\alpha-1} \lambda f(s, u(s)) d s\right)=0 \\
u^{(i)}(0)=0, i=0,1, \ldots, n-1 .
\end{array}\right. \\
& \text { Now, equation e-ID-1 yields } \\
& \mathrm{u}(t)=\frac{1}{\Gamma(\beta)} \int_{0}^{t}(t-s)^{\beta-1} \phi_{q}\left(\frac{1}{\Gamma(\alpha)} \int_{s}^{1}(r-s)^{\alpha-1} \lambda f(r, u(r)) d r\right) d s .
\end{aligned}
$$

From which we obtain the required result.

Define the integral operator $T: E \rightarrow E$ by

$\operatorname{Tu}(t)=\frac{1}{\Gamma(\beta)} \int_{0}^{t}(t-s)^{\beta-1} \phi_{q}\left(\frac{1}{\Gamma(\alpha)} \int_{s}^{1}(r-s)^{\alpha-1} \lambda f(r, u(r)) d r\right) d s$, where the Banach space $E=C[0,1]$ is endowed with the norm

$\|u\|=t \in[0,1] \max |u(t)|$.

Now, we are in a position to establish the existence of nontrivial solution for the fractional boundary value problem 1.1. 
Theorem 3. Suppose that $f(t, 0) \neq 0$ and there exist nonnegative functions $h \in L^{1}[0,1]$ and $\psi \in C[0,+\infty)$, with $\psi$ nondecreasing, such that

$|f(t, x)| \leq h(t) \psi(|x|)$. Then problem 1.1 has at least one nontrivial solution for all $\lambda>0$.

Proof. We are going to use the Schaefer's fixed point Theorem to prove the result. So fix $\lambda>0$. The following properties hold

(i) $T$ maps bounded sets into bounded sets. Let $u \in B_{\delta}=\{u \in E,\|u\|<\delta\}$, $\delta>0$, then we have

$$
\begin{aligned}
|T u(t)| & \leq \frac{1}{(\Gamma(\alpha))^{q-1} \Gamma(\beta)} \int_{0}^{t}(t-s)^{\beta-1} \phi_{q}\left(\int_{s}^{1}(r-s)^{\alpha-1}|\lambda f(r, u(r))| d r\right) d s \\
& \leq \frac{\lambda^{q-1}}{(\Gamma(\alpha))^{q-1} \Gamma(\beta)}\left(\int_{0}^{1} h(r) \psi(|u(r)|) d r\right)^{q-1} \\
& \leq \frac{\left(\lambda \psi(\delta)\|h\|_{L^{1}[0,1]}\right)^{q-1}}{(\Gamma(\alpha))^{q-1} \Gamma(\beta)}<+\infty .
\end{aligned}
$$

Hence, $T\left(B_{\delta}\right)$ is uniformly bounded.

(ii) $T\left(B_{\delta}\right)$ is equicontinuous. For $0 \leq$ $\mathrm{t}_{1}<t_{2} \leq 1$, we have

$$
\begin{aligned}
& \left|T u\left(t_{2}\right)-T u\left(t_{1}\right)\right| \\
\leq & \frac{1}{(\Gamma(\alpha))^{q-1} \Gamma(\beta)}\left(\int_{0}^{t_{1}}\left(\left(t_{2}-s\right)^{\beta-1}-\left(t_{1}-s\right)^{\beta-1}\right) \phi_{q}\right) \\
& \left(\int_{s}^{1}(r-s)^{\alpha-1}|\lambda f(r, u(r))| d r\right) d s \\
& \left.+\int_{t_{1}}^{t_{2}}\left(t_{2}-s\right)^{\beta-1} \phi_{q}\left(\int_{s}^{1}(r-s)^{\alpha-1}|\lambda f(r, u(r))| d r\right) d s\right) \\
\leq & \frac{\left(\lambda \psi(\delta)\|h\|_{L^{1}[0,1]}\right)^{q-1}}{(\Gamma(\alpha))^{q-1} \Gamma(\beta)}\left(\int_{0}^{t_{1}}\left(\left(t_{2}-s\right)^{\beta-1}-\left(t_{1}-s\right)^{\beta-1}\right) d s\right. \\
& \left.+\int_{t_{1}}^{t_{2}}\left(t_{2}-s\right)^{\beta-1} d s\right) \rightarrow 0, \text { as } t_{1} \rightarrow t_{2} .
\end{aligned}
$$

From Arzelà-Ascoli Theorem, we deduce that the operator $T$ is compact. 
(iii) Let $\Omega=B_{\delta}, \delta>0$, then for $u \in \Omega$, such that $u=\mu T u, 0<\mu<1$, we obtain

$|u(t)| \leq|T u(t)| \leq \frac{\left(\lambda \psi(\delta)\|h\|_{L^{1}[0,1]}\right)^{q-1}}{(\Gamma(\alpha))^{q-1} \Gamma(\beta)}$, which implies that the set of solutions is uniformly bounded.

So, all assumptions of Schaefer's Theorem are fulfilled, consequently $T$ has a fixed point in $\bar{\Omega}$ which, since $f(t, 0) \not \equiv 0$, is a nontrivial solution of problem 1.1 in $E$.

\section{Lyapunov inequality}

In this section, we are going to establish the Lyapunov inequality for the fractional problem 1.2

Theorem 1. (Lyapunov type inequality). Let $u$ be a nontrivial solution of problem 1.2, then the inequality $\int_{a}^{b}|\chi(r)| d r \geq \frac{\Gamma(\alpha+1)(\Gamma(\beta))^{p-1}}{(b-a)^{(p-1) \beta+\alpha-1}}\left(\frac{\beta(p-1)-1}{p-2}\right)^{p-2}$, holds, where $p>2$ and $(p-1) \beta \geq 1$.

Proof. From the properties of the Caputo derivative showed in Proposition 4 and the boundary conditions $D_{a^{+}}^{\beta+i} u(b)=0, i=0,1, \ldots, n-1$, we obtain

$$
I_{b^{-}}^{\alpha C} D_{b^{-}}^{\alpha}\left(\phi_{p}\left(D_{0^{+}}^{\beta} u(t)\right)\right)=\phi_{p}\left(D_{0^{+}}^{\beta} u(t)\right) .
$$

Now, by applying the operator $I_{b^{-}}^{\alpha}$ on both sides of the differential equation in 1.2 and using (4.1), we get

$\phi_{p}\left(D_{0^{+}}^{\beta} u(t)\right)=\frac{1}{\Gamma(\alpha)} \int_{t}^{b}(s-t)^{\alpha-1} \chi(s) \phi_{p}(u(s)) d s$, thus, we have

$$
\left|\left(D_{0^{+}}^{\beta} u(t)\right)\right|^{p-1} \leq \frac{1}{\Gamma(\alpha)} \int_{t}^{b}|\chi(s)||u(s)|^{p-1}(s-t)^{\alpha-1} d s .
$$

Also, from the boundary conditions $u^{(i)}(a)=0, i=0,1, \ldots, n-1$, yields $\mathrm{u}(t)=I_{a^{+}}^{\beta} D_{a^{+}}^{\beta} u(t)$, so, we have $|u(t)| \leq \frac{1}{\Gamma(\beta)} \int_{a}^{t}(t-s)^{\beta-1}\left|D_{0^{+}}^{\beta} u(s)\right| d s$. By using Hölder inequality on the integral of the right-hand side of the above inequality, we obtain

$$
\begin{aligned}
|u(t)| & \leq \frac{1}{\Gamma(\beta)}\left(\int_{a}^{t}(t-s)^{\frac{(\beta-1)(p-1)}{p-2}} d s\right)^{\frac{p-2}{p-1}}\left(\int_{a}^{t}\left|D_{a^{+}}^{\beta} u(s)\right|^{p-1} d s\right)^{\frac{1}{p-1}} \\
& =\frac{1}{\Gamma(\beta)}\left(\frac{p-2}{\beta(p-1)-1}(t-a)^{\frac{(p-1) \beta-1}{p-2}}\right)^{\frac{p-2}{p-1}}\left(\int_{a}^{t}\left|D_{a^{+}}^{\beta} u(s)\right|^{p-1} d s\right)^{\frac{1}{p-1}}
\end{aligned}
$$


then,

$|u(t)|^{p-1} \leq \frac{1}{(\Gamma(\beta))^{p-1}}\left(\frac{p-2}{\beta(p-1)-1}(t-a)^{\frac{(p-1) \beta-1}{p-2}}\right)^{p-2} \int_{a}^{t}\left|D_{a^{+}}^{\beta} u(s)\right|^{p-1} d s$.

Substituting (4.2) in (4.3) yields

$|u(t)|^{p-1} \leq \frac{1}{\Gamma(\alpha)(\Gamma(\beta))^{p-1}}\left(\frac{p-2}{\beta(p-1)-1}(t-a)^{\frac{(p-1) \beta-1}{p-2}}\right)^{p-2}$

$\int_{a}^{t} \int_{s}^{b}|\chi(r)||u(r)|^{p-1}(r-s)^{\alpha-1} d r d s$,

then, by interchanging the order of integration, we get

$$
\begin{aligned}
|u(t)|^{p-1} \leq & \frac{1}{\Gamma(\alpha)(\Gamma(\beta))^{p-1}}\left(\frac{p-2}{\beta(p-1)-1}(t-a)^{\frac{(p-1) \beta-1}{p-2}}\right)^{p-2} \\
& \left(\int_{a}^{t}|\chi(r)||u(r)|^{p-1} \int_{a}^{r}(r-s)^{\alpha-1} d s d r\right. \\
& \left.+\int_{t}^{b}|\chi(r)||u(r)|^{p-1} \int_{a}^{t}(r-s)^{\alpha-1} d s d r\right) \\
= & \frac{1}{\Gamma(\alpha)(\Gamma(\beta))^{p-1}}\left(\frac{p-2}{\beta(p-1)-1}(t-a)^{\frac{(p-1) \beta-1}{p-2}}\right)^{p-2} \\
& \int_{a}^{t}|\chi(r)||u(r)|^{p-1} \frac{(r-a)^{\alpha}}{\alpha} d r \\
& \left.+\int_{t}^{b}|\chi(r)||u(r)|^{p-1} \frac{(r-a)^{\alpha}-(r-t)^{\alpha}}{\alpha} d r\right) \\
\leq & \frac{1}{\Gamma(\alpha+1)(\Gamma(\beta))^{p-1}}\left(\frac{p-2}{\beta(p-1)-1}(t-a)^{\frac{(p-1) \beta-1}{p-2}}\right)^{p-2} \\
& \int_{a}^{b}|\chi(r)||u(r)|^{p-1}(r-a)^{\alpha} d r .
\end{aligned}
$$

Therefore, we obtain $\|u\|^{p-1} \leq \frac{1}{\Gamma(\alpha+1)(\Gamma(\beta))^{p-1}}\left(\frac{p-2}{\beta(p-1)-1}(b-a)^{\frac{(p-1) \beta-1}{p-2}}\right)^{p-2}$ $\int_{a}^{b}|\chi(r)|\|u\|^{p-1}(b-a)^{\alpha} d r$. Thus

$1 \leq \frac{(b-a)^{(p-1) \beta+\alpha-1}}{\Gamma(\alpha+1)(\Gamma(\beta))^{p-1}}\left(\frac{p-2}{\beta(p-1)-1}\right)^{p-2} \int_{a}^{b}|\chi(r)| d r$, which yields the required result.

Example 2. Consider the following problem

$$
\left\{\begin{array}{c}
-{ }^{C} D_{1^{-}}^{\frac{3}{2}}\left(\phi_{p}\left(D_{0^{+}}^{\frac{7}{4}} u(t)\right)\right)+\lambda f(t, u(t))=0,0<t<1 \\
u(0)=u^{\prime}(0)=D_{0^{+}}^{\frac{7}{4}} u(1)=D_{0^{+}}^{\frac{11}{4}} u(1)=0,
\end{array}\right.
$$


where $f(t, x)=\frac{1+\arctan x}{\sqrt{1+t}}$.

Obviosly, we have $f(t, 0)=\frac{1}{\sqrt{1+t}}=0$ and

$|f(t, x)| \leq h(t) \psi(|x|)$, where $h(t)=\frac{1}{\sqrt{1+t}} \in L^{1}[0,1]$ and $\psi(x)=1+$ $\arctan x$.

From Theorem 8 we conclude that for all $\lambda>0$, this problem admits at least one solution.

\section{References}

[1] B. Ahmad, S.K. Ntouyas, and A. Alsaedi, "Existence theory for nonlocal boundary value problems involving mixed fractional derivatives", Nonlinear analysis: modelling and control, vol. 24, no. 6, pp. 937-957, 2019, doi: 10.15388/ NA.2019.6.6

[2] B. Ahmad, A. Broom, A. Alsaedi, and S. K. Ntouyas, "Nonlinear integrodifferential equations involving mixed right and left fractional derivatives and integrals with nonlocal boundary data", Mathematics, vol. 8, no. 3, Art ID. 336, 2020, doi: 10.3390/ math8030336

[3] B. Ahmad, S. K. Ntouyas, and A. Alsaedi, "Fractional order differential systems involving right Caputo and left Riemann-Liouville fractional derivatives with nonlocal coupled conditions", Boundary value problems, vol. 2019, no. 1, Art ID. 109, 2019, doi: 10.1186/s13661019-1222-0

[4] R. Almeida, S. Pooseh, and D. F. M. Torres, Computational methods in the fractional calculus of variations. London: Imperial College, 2015, doi: $10.1142 /$ p991

[5] A. Alsaedi, A. Broom, S. K. Ntouyas, and B. Ahmad, "Nonlocal fractional boundary value problems involving mixed right and left fractional derivatives and integrals", Axioms, vol. 9, no. 2, Art ID. 50, 2020, doi: 10.3390/axioms9020050

[6] A. Guezane-Lakoud, R. Khaldi, and A. Kılıçman, "Existence of solutions for a mixed fractional boundary value problem", Advances in difference equations, Art. ID. 164 2017, doi: 10.1186/ s13662-017-1226-y

[7] A. Guezane-Lakoud, R. Khaldi, and D. F.M. Torres, "Lyapunov-type inequality for a fractional boundary value problem with natural conditions", SeMA journal, vol. 75, pp. 157-162, 2018, doi: 10.1007/s40324-017-0124-2 
[8] A. Guezane-Lakoud, R. Khaldi, and D. F. M. Torres, "On a fractional oscillator equation with natural boundary conditions", Progress in fractional differentiation and applications, vol. 3, no. 3, pp. 191-197, 2017, doi: 10.18576/ pfda/ 030302

[9] R. Khaldi and A Guezane-Lakoud, "On a generalized Lyapunov inequality for a mixed fractional boundary value problem", AIMS mathematics, vol. 4, no. 3, pp. 506-515, 2019, doi: 10.3934/math.2019.3.506

[10] R. Khaldi and A Guezane-Lakoud, "Lyapunov inequality for a boundary value problem involving conformable derivative", Progress in fractional differentiation and applications, vol. 4, no. 4, pp. 323-329, doi: $10.18576 /$ pfda/ 030407

[11] R. Khaldi and A. Guezane-Lakoud, "Higher order fractional boundary value problems for mixed type derivatives", Journal of nonlinear functional analysis, vol. 2017, Art. ID 30, 2017, doi: 10.23952/jnfa.2017.30

[12] A. A. Kilbas, H. M. Srivastava, and J. J. Trujillo, Theory and applications of fractional differential equations. Amsterdam: Elsevier Science, 2006.

[13] A. B. Malinowska, T. Odzijewicz, and D. F. M. Torres, Advanced methods in the fractional calculus of variations. Cham: Springer, 2015, doi: 10.1007/978-3-319-14756-7

[14] A. B. Malinowska and D. F. M. Torres, "Generalized natural boundary conditions for fractional variational problems in terms of the Caputo derivative", Computers \& mathematics with applications, vol. 59, no. 9, pp. 3110-3116, 2010, doi: 10.1016/j.camwa.2010.02.032

[15] I. Merzoug, A. Guezane-Lakoud, and R. Khaldi, "Existence of solutions for a nonlinear fractional p-Laplacian boundary value problem", Rendiconti del Circolo Matematico di Palermo Series 2, vol. 69, 2019, doi: 10.1007/ s12215-019-00459-4

[16] S. K. Ntouyas, B. A. Ahmad, and T. P. Horikis, "Recent developments of Lyapunov-type inequalities for fractional differential equations", in Differential and integral inequalities, D. Andrica and T. Rassias, Eds. Cham: Springer, 2019, pp. 619-686, doi: 10.1007/978-3-030-27407 $-824$

[17] I. Podlubny, Fractional differential equations. San Diego, CA: Academic Press, 1999. 
[18] S. G. Samko, O. I. Marichev, and A. A. Kilbas, Fractional integrals and derivatives: theory and applications. Amsterdam: Gordon and Breach Science Publishers, 1993 [Online]. Available: https:/ / bit.ly/ 2WNwdlk

[19] E. Zeidler, Nonlinear functional analysis and its applications, vol. 1. New York, NY: Springer, 1986. 